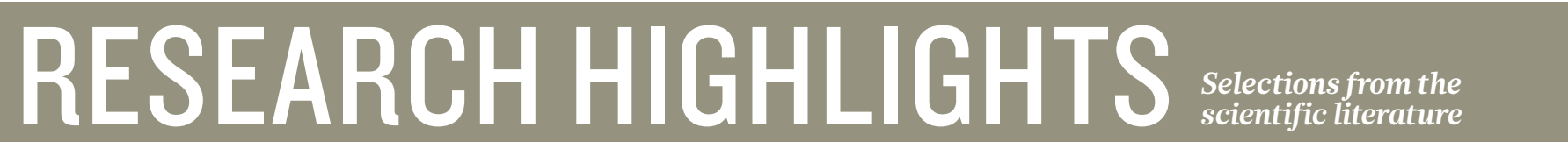

ECOLOGY

\section{Frogs at home with relatives}

Charles Darwin proposed that competition would lower the success of species invasions if an ecosystem contained closely related species. But he may have been wrong, at least where amphibians are concerned. Members of this class are more likely to successfully establish themselves in new environments when they are related to species already resident there.

Reid Tingley and his colleagues at the University of Sydney, Australia, analysed data on 521 successful and failed introductions of 162 amphibian species. They found that the chances of successful invasion rose as the richness of related species grew.

The results could aid conservation efforts by helping to predict whether introduced species will be successful in establishing themselves.

Am. Nat. doi:10.1086/658342 (2011)

MATERIALS SCIENCE

\section{Chemical scissors cut graphene}

High-performance electronic devices made entirely of carbon could be created using low-cost, conventional microfabrication approaches to pattern one-atom-thick

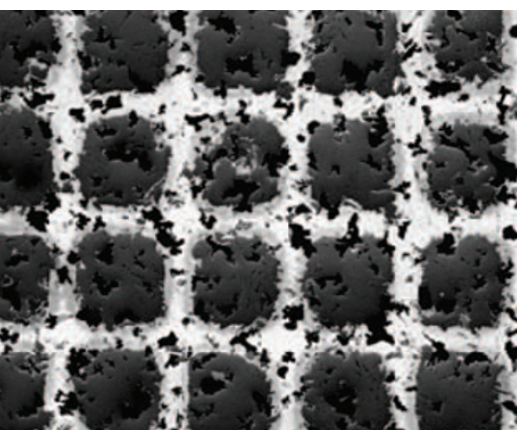

layers of carbon called graphene.

Zhongfan Liu at Peking University in Beijing and his collaborators laid a titanium dioxide-based photomask - a small stencil - on a sheet of graphene. The photomask catalyses the formation of hydroxyl radicals when ultraviolet light is shone onto it. This highly reactive species oxidizes graphene only in the irradiated regions - essentially acting like scissors to break carbon bonds and produce patterns (pictured). Using this approach, the authors fabricated an all-carbon transistor that performed similarly to conventional ones. The method is solvent-free, so avoids possible contamination issues.

J. Am. Chem. Soc. doi:10.1021/ ja109934b (2011)

\section{INFECTIOUS DISEASES}

\section{Targeting typhoid fever}

Many thousands of people around the world die of typhoid fever every year, but little is known about how the bacterium Salmonella enterica Typhi causes the disease. A protein identified on the microbe's outer surface seems to be key to $S$. enterica Typhi's virulence and could be a target for more effective vaccines.

Using bioinformatic techniques, Santasabuj Das and his colleagues at the National Institute of Cholera and Enteric Diseases in Kolkata, India, looked for S. enterica Typhi proteins that could bind to host receptors and homed in on one, called T2544. Bacteria in which the gene for this protein had been deleted showed reduced adhesion to human cells and, in particular, to laminin, a protein abundant in the matrix

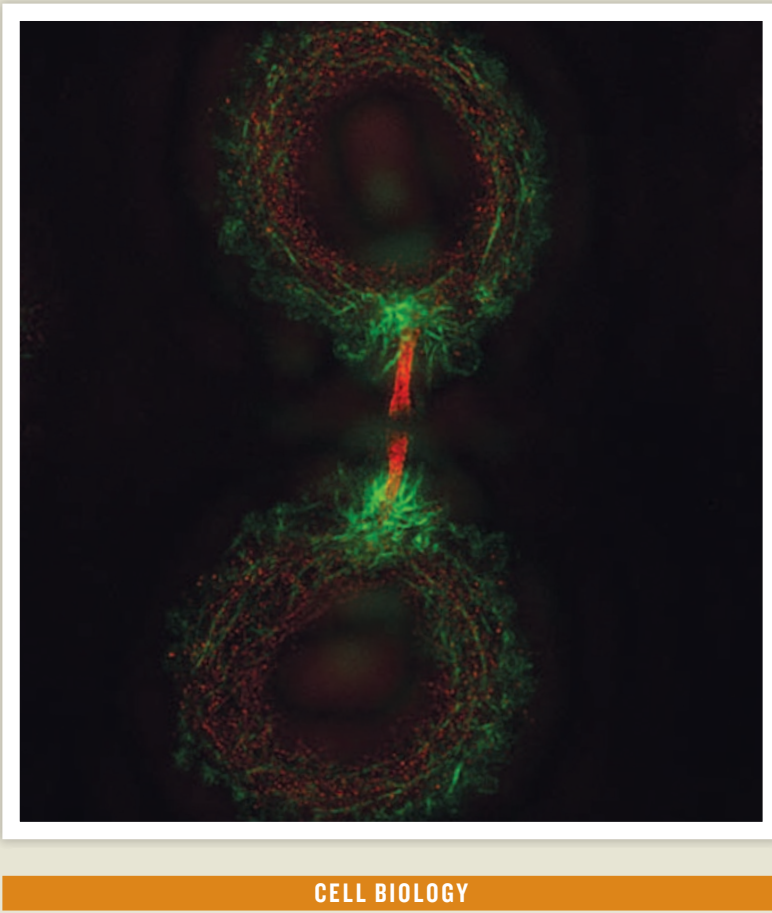

\section{Cell division in a pinch}

Time-lapse images have revealed the final steps of cell division, when a thin intercellular bridge connecting the two cells (pictured) splits.

Daniel Gerlich at the Swiss Federal Institute of Technology in Zurich and Thomas Müller-Reichert at Dresden University of Technology in Germany found that helical protein filaments wrap around and constrict this bridge, narrowing it to a single stalk just before the cells separate.

High-resolution imaging of a fluorescently tagged protein found in a complex called ESCRT-III revealed that the complex accumulates where the bridge tightens. In cells lacking the complex, the intercellular bridge did not constrict. The authors propose that ESCRT-III components join together to form the filament helices.

Science doi:10.1126/science.1201847 (2011)

between cells. When mice were infected with a specific dose of the normal bacteria, half died within a week, whereas animals infected with the same dose of the mutant strain all survived. Moreover, T2544 triggered a protective antibody response in the mice.

Proc. Natl Acad. Sci. USA doi:10.1073/pnas.1016180108 (2011)

\section{PALAEOCLIMATE \\ Ice-smothered British isles}

Ice sheets repeatedly crept down to cover extensive areas of Britain and Ireland at the start of the Northern Hemisphere ice age - more than 1 million years earlier than previously thought. 